Research Paper

\title{
Biodegradable Nitrogen-Doped Carbon Nanodots for Non-Invasive Photoacoustic Imaging and Photothermal Therapy
}

\author{
Changho Lee ${ }^{1,5 *}$, Woosung Kwon ${ }^{2 *}$, Songeun Beack ${ }^{3 *}$, Donghyun Lee ${ }^{1}$, Yoonsang Park ${ }^{4}$, Hyemin Kim ${ }^{3}$, Sei \\ Kwang Hahn ${ }^{3 凶}$, Shi-Woo Rhee $2,4 \bowtie$, Chulhong Kim ${ }^{1 凶}$ \\ 1. Department of Creative IT Engineering, Pohang University of Science and Technology (POSTECH), 77 Cheongam-ro, Pohang 37673, South Korea; \\ 2. Department of Chemical and Biological Engineering, Sookmyung Women's University, 100 Cheongpa-ro 47-gil, Seoul 04310, South Korea; \\ 3. Department of Materials and Science Engineering, Pohang University of Science and Technology (POSTECH), 77 Cheongam-ro, Pohang 37673, South Korea; \\ 4. Department of Chemical Engineering, Pohang University of Science and Technology (POSTECH), 77 Cheongam-ro, Pohang 37673, South Korea. \\ 5. Current address: Department of Electrical and Computer Engineering, Johns Hopkins University, 3400 North Charles Street, Baltimore, MD 21218, USA. \\ * These authors contributed equally on this work. \\ $\square$ Corresponding authors: E-mail: chulhong@postech.edu (Phone, +82-54-279-8805; Fax, 82-54-279-8899), skhanb@postech.edu (Phone, +82-54-279-2159; Fax, \\ 82-54-279-2399), and srhee@sookmyung.ac.kr (Phone, +82-02-2077-7809; Fax, 82-02-2077-7450).
}

() Ivyspring International Publisher. Reproduction is permitted for personal, noncommercial use, provided that the article is in whole, unmodified, and properly cited. See http://ivyspring.com/terms for terms and conditions.

Received: 2016.07.21; Accepted: 2016.08.20; Published: 2016.09.22

\begin{abstract}
Multifunctional nanoparticles have been widely investigated for biomedical applications, such as imaging, therapy, and drug delivery. Especially, photoactive nanoparticles have received great attention as theranostic agents because of their heat-generating abilities after exposure to laser irradiation. However, photostability and safety issues have been the technical hurdles for further clinical applications. Here, we designed nitrogen (N)-doped carbon nanodots (N-CNDs) that have strong absorption in the near-infrared region, high photostability, and excellent biodegradability. Optimized N-CNDs can be utilized not only as a new photoacoustic (PA) imaging agent but also as a superior photothermal therapy (PTT) agent in vivo because of their strong optical absorption at a specific wavelength. We used $\mathrm{N}-\mathrm{CNDs}$ to perform in vivo/ex vivo noninvasive PA imaging of sentinel lymph nodes via local delivery and performed PTT for cancer ablation therapy. Finally, biodegradation and renal clearance were confirmed by performing whole-body PA monitoring and a degradation test.
\end{abstract}

Key words: Carbon nanodot, Nitrogen doping, Photoacoustic, Photothermal, Biodegradation.

\section{Introduction}

There have been significant achievements in the development of multifunctional nanoparticles, which have stimulated various biomedical research areas, including molecular imaging, cancer therapy, and drug delivery [1-5]. In particular, photoactive nanoparticles have been widely used as diagnostic and therapeutic agents with laser irradiation [6, 7]. When photoactive nanoparticles are irradiated with nanosecond pulsed laser light, they heat up and generate acoustic waves that can be used for photoacoustic (PA) imaging. PA imaging is regarded as an emerging non-invasive imaging technique that provides high ultrasonic resolution and strong, optically sensitive images in deep tissues because of a laser-induced PA effect [8-10]. Because of its hybrid properties, the PA imaging technique provides in vivo deep tissue high-resolution anatomical, functional, and molecular images [11-19]. In another study, continuous-wave laser irradiation of a photo-activated nanoparticle-injected region was used to generate a temperature increase in a selected area; this technique was used as photothermal therapy (PTT) for tumor ablation. PTT has received attention because it facilitates selective tumor ablation 
and minimizes damage to normal tissues [20-23]. Therefore, it could be advantageous to combine contrast agent-based PA imaging and PTT within a single probe for use in disease diagnosis and therapy.

Among the various types of nanoparticles, metallic nanoparticles, such as gold-based nanostructures [2, 24-29], carbon-based nanoparticles $[25,30,31]$, and organic nanoparticles [32-36], have been reported as multifunctional nanoparticles for PA imaging and PTT because of their strong photo-absorbing abilities. Unfortunately, even for the metallic nanoparticles that have shown excellent performance, their photostability, biocompatibility, and biodegradability remain critical issues for true clinical translation. For example, long-term laser irradiation of gold nanoparticles can cause morphological changes and will generally cause changes in the optical properties of the nanoparticles and reduce absorbance [37, 38]. Additionally, gold nanoshells coated with polyethylene glycol 5000 have been shown to be difficult to excrete from the body [39], and the use of surfactants during gold nanorod synthesis has been shown to cause toxicity [40,41]. Thus, alternative agents with strong absorbance must maintain the optical stability and biodegradability of the nanoparticles.

Carbon nanoparticles are regarded as being relatively more stable and biocompatible than are the nanostructures described above [42, 43]. Carbon nanodots (CNDs) are promising carbon-based imaging probes that have attracted much attention because of their properties, including excellent biocompatibility, water solubility, and photostability [44-51]. Recently, several studies have successfully demonstrated the feasibility of using CNDs as imaging probes [52-57], but the main challenge for practical applications is to guarantee biodegradability. Furthermore, the fundamental mechanism of how CNDs interact with low-energy photons should be studied to improve their optical properties related to image resolution and contrast.

In this study, we synthesized biocompatible, near-infrared (NIR)-absorbing N-doped CNDs (N-CNDs) for PA imaging and PTT by carbonizing organic acids with a controllable nitrogen source. The synthesized N-CNDs showed excellent photostability and strong optical absorbance in the NIR region, where tissue and hemoglobin show small optical absorption. Thus, the PA signals from N-CNDs were high enough to detect inside living animals, and minimally invasive PTT using N-CNDs was possible. We performed time-resolved PA imaging of sentinel lymph nodes (SLNs) and assessed renal clearance after hypodermic injection to investigate the biodegradability and potential application of N-CNDs as a PA imaging contrast agent. Degradation and cytotoxic tests were also performed to further verify the biodegradability of N-CNDs. Finally, we treated the liver cancer tumor on the mouse skin using photothermal ablation with N-CNDs injected near the tumor region.

\section{Materials and Methods}

\subsection{Synthesis of N-CNDs}

An aqueous solution of citric acid was prepared by dissolving citric acid $(1 \mathrm{~g})$ in water $(1 \mathrm{~mL})$. The aqueous solution of citric acid was transferred into a round-bottom flask containing oleylamine $(1 \mathrm{~mL})$ and octadecene $(9 \mathrm{~mL})$. To the mixture under vigorous stirring, $0,1,3,5,7,10,12$, and $14 \mathrm{M}$ (conc.) solutions of nitric acid $(1 \mathrm{~mL})$ were added for synthesizing N0-, N1-, N3-, N5-, N7-, N10-, N12-, and N14-CNDs, respectively. The mixture was then heated for $3 \mathrm{~h}$ at $250^{\circ} \mathrm{C}$. The resulting solution $(5 \mathrm{~mL})$ was mixed with methanol $(40 \mathrm{~mL})$ and then separated by centrifugation at 3,000 rpm for $15 \mathrm{~min}$, which was repeated three times. After drying in a vacuum oven overnight, the resulting viscous liquid $(50 \mathrm{mg})$ was re-dispersed in toluene $(5 \mathrm{~mL})$, and to this solution, ethanolamine $(15 \mathrm{~mL})$ was added. The mixture was then heated at $100^{\circ} \mathrm{C}$ under vigorous stirring until solid remained. The resulting solid was dissolved in water $(10 \mathrm{~mL})$ and then dialyzed against water three times (24 h for each time) using Spectra/Por Biotech Cellulose Ester dialysis tubes (100-500 Da). The water was removed from the resulting solution by freeze-drying. The resulting solid (N-CNDs) was stored in a freezer for further use.

\subsection{PA imaging system}

All in vitro, in vivo, and ex vivo PA experiments were conducted using an acoustic-resolution reflection-mode PA imaging system (Figure S1). A Q-switched Nd:YAG laser (SLII-10; Continuum) at 532-nm optical wavelength was used for pumping a tunable OPO laser (Surelite OPOPLUS; Continuum), which provided a 5-ns pulse duration over the wavelength tuning range of 680 to $2500 \mathrm{~nm}$ at a $10-\mathrm{Hz}$ repetition rate. To avoid PA signal generation from the surface, the irradiated laser beam had a donut-shaped pattern achieved by passing through a conical lens and an optical condenser. The pulsed laser energy was $\sim 5 \mathrm{~mJ} / \mathrm{cm}^{2}$, which is much less than the American National Standards Institute standard limit $\left(\sim 20 \mathrm{~mJ} / \mathrm{cm}^{2}\right)$. A wavelength of $680 \mathrm{~nm}$ was used for in vivo PA imaging. A spherically focused ultrasonic transducer with a 5-MHz center frequency (V308; Olympus NDT) was applied for detecting the generated PA signals. A pulser/receiver (5072PR, Olympus NDT) and a digital oscilloscope (MSO 5204; 
Tektronix) were used for amplifying the PA signal and recoding, respectively. Two linear scanners were used to perform raster scanning to obtain volumetric PA imaging. To improve the acoustic coupling efficiency, a homemade water tank and ultrasound gel were used. The animals were then placed on a sample stage for SLN and bladder imaging. The 5-MHz ultrasonic transducer induced axial and lateral resolutions of 145 and $950 \mu \mathrm{m}$, respectively. It takes approximately $30 \mathrm{mins}$ to acquire one three-dimensional PA image.

\subsection{In vitro/in vivo/ex vivo PA imaging setup}

To measure the in vitro PA sensitivity and spectrum of N-CNDs, eight silicon tubes were prepared with a N0-, N1-, N3-, N5-, N7-, N10-, N12-, and N14-CNDs at the same concentration (20 $\mathrm{mg} / \mathrm{mL}$ ) by tuning the laser wavelength from 680 to $950 \mathrm{~nm}$. Moreover, to compare the PA amplitude of $\mathrm{N}$-CNDs with those of conventional materials at the same optical density of $0.6 \mathrm{~mm}^{-1}$, we measured the PA signal amplitudes of three silicon tubes containing gold nanorod (GNR), methylene blue (MB), and $\mathrm{N}-\mathrm{CNDs}$ under 680-nm pulsed laser excitation. Furthermore, to investigate the stability of the PA response, GNR, $\mathrm{MB}$, and $\mathrm{N}-\mathrm{CNDs}(20 \mathrm{mg} / \mathrm{mL})$ were also compared by monitoring the PA amplitudes during 1,100 pulsed laser shots of $15 \mathrm{~mJ} / \mathrm{cm}^{2}$.

We satisfied the guidelines of the university on the care and utilization of laboratory animals in all animal experiments. For in vivo and ex vivo SLN PA imaging, female Sprague-Dawley rats (weight: $\sim 250$ g) were fully anesthetized by using a vaporized-isoflurane system. After removing the hair of the left-side axillae, the rat was placed on the sample stage. The left-side axillae vasculature networks and lymphatic system of the rats before and after hypodermic injection of N-CNDs $(0.1 \mathrm{~mL}, 20$ $\mathrm{mg} / \mathrm{mL})(\mathrm{n}=3)$ were photoacoustically visualized. After completing in vivo PA imaging, we performed validation by performing ex vivo PA imaging of extracted lymph nodes. For in vivo whole-body PA imaging, female Balb/c nu/nu mice (Weight: 20 g) were prepared under full anesthetization. After obtaining control whole-body PA images of the abdomen side with an invisible bladder, the N-CNDs $(0.1 \mathrm{~mL}, 20 \mathrm{mg} / \mathrm{mL})(\mathrm{n}=3)$ were subcutaneously delivered into the left leg. Then, we photoacoustically monitored the visualization of the bladder over 280 min.

\subsection{PTT materials}

Dulbecco's modified Eagle's medium (DMEM), fetal bovine serum (FBS), antibiotics, and phosphate-buffered solution (PBS) were purchased from Invitrogen (Carlsbad, CA). 3-(4,5-Dimethyl-2thiazolyl)-2,5-diphenyl-2H-tetrazolium bromide (MTT) was supplied from Sigma-Aldrich (St. Louis, $\mathrm{MO}$ ). Calcein-AM and propidium iodide (PI) were obtained from AnaSpec (Fremont, CA). The 8-chamber glass slides with polystyrene vessels were purchased from BD Falcon (Franklin Lakes, NJ).

\subsection{Photothermal performance of N-CNDs}

$\mathrm{N}-\mathrm{CNDs}$ were dissolved in PBS at different concentrations (0 $\mathrm{mg} / \mathrm{mL}$ to $20 \mathrm{mg} / \mathrm{mL})$. Each $\mathrm{N}-\mathrm{CNDs}$ solution $(1 \mathrm{~mL})$ was introduced in a quartz cuvette and irradiated with $808-\mathrm{nm}$ laser light at a power density of $2 \mathrm{~W} / \mathrm{cm}^{2}$ for $10 \mathrm{~min}$. To observe the photothermal characteristics of N-CNDs, a thermocouple probe was inserted into the cuvette to contact the N-CNDs solution, and the temperature was recorded every $30 \mathrm{~s}$. PBS was used as a negative control.

\subsection{Confocal microscopy for cellular uptake}

Normal mouse liver hepatocyte cells (FL83B) and human liver cancer cells (HepG2) were cultured in high-glucose DMEM supplemented with $10 \mathrm{vol} \%$ of FBS at $37^{\circ} \mathrm{C}$ in an incubator. The cells were seeded in the 8-chamber confocal slide at a density of $4 \times 10^{4}$ cells/well and incubated for a day. Then, N-CNDs solutions $(10 \mathrm{mg} / \mathrm{mL})$ in $200 \mu \mathrm{L}$ of DMEM were added to the wells. The cellular uptake of N-CNDs was assessed by confocal microscopy. The emission light of N-CNDs was spectrally resolved into four channels. We observed strong blue, green, and yellow fluorescence from N-CNDs-labeled cells depending on the excitation wavelength, and less fluorescence was observed from the control group.

\subsection{Cytotoxic test}

FL83B cells and HepG2 cells were seeded in a 96-well plate at $1 \times 10^{4}$ cells/well and cultured in a humidified $5 \% \mathrm{CO}_{2}$ incubator at $37^{\circ} \mathrm{C}$ for $24 \mathrm{~h}$. $\mathrm{N}-\mathrm{CNDs}$ were dissolved in a cell culture medium containing $10 \mathrm{vol} \%$ FBS and $1 \mathrm{wt} \%$ of antibiotics. The cells were allowed to incubate with different concentrations of N-CNDs (0, 0.1, 0.25, 0.5, 1, 2.5, 5, and $10 \mathrm{mg} / \mathrm{mL}$ ) for another $24 \mathrm{~h}$ at $37^{\circ} \mathrm{C}$, respectively. After the cells were washed twice with PBS, the cell culture medium was changed. The relative cell viability was measured by MTT assay $(n=3)$. The absorbance was measured at $540 \mathrm{~nm}$.

\subsection{Photothermal effect of N-CNDs in vitro}

HepG2 cells at a density of $1 \times 10^{4}$ were seeded onto 96-well plates. After incubation for $24 \mathrm{~h}$, the cells were treated with different concentrations of N-CNDs $(0,0.1,1$, and $10 \mathrm{mg} / \mathrm{mL})$ for 1 hour. After 10-min exposure of $808-\mathrm{nm}$ light at $2 \mathrm{~W} / \mathrm{cm}^{2}$, the cells were 
allowed to incubate for an additional $4 \mathrm{~h}$. Cells treated without laser were used as a control group. To assess the photothermal effect of N-CNDs on the cells with and without exposure to NIR laser irradiation, the phototoxicity was measured by MTT assay $(n=3)$. To further evaluate the efficacy of PTT, HepG2 cells at a density of $3 \times 10^{4}$ were incubated with and without $\mathrm{N}-\mathrm{CNDs}(10 \mathrm{mg} / \mathrm{mL})$ for $12 \mathrm{~h}$ and then irradiated with 808-nm laser light $\left(2 \mathrm{~W} / \mathrm{cm}^{2}\right)$ for $10 \mathrm{~min}$. After another incubation for $2 \mathrm{~h}$, the cells were washed with PBS, stained with calcein-AM and PI, and then imaged by Leica TCS SP5II MP SMD FLIM (Leica, Deerfield, IL). The excitation laser was tuned to 488 and $594 \mathrm{~nm}$.

\subsection{Photothermal effect of N-CNDs in vivo}

Animal experiments were approved by the university in compliance with the regulations concerning experimental animals. To assess the therapeutic efficacy of N-CNDs, cancer model mice were prepared by inoculation with HepG2 cells at a density of $1 \times 10^{7}$ to the left and right flanks of female Balb/c nude mice (5 weeks old, 20-25 g) and allowed to grow for 3-5 days until the tumor volume reached approximately $27 \mathrm{~mm}^{3}(\mathrm{n}=5)$. The tumor was treated by intratumoral injection of the solutions of PBS and N-CNDs $(100 \mu \mathrm{L}, 20 \mathrm{mg} / \mathrm{mL})$ through a 26-gauge needle. The right tumors only were irradiated with $808-\mathrm{nm}$ laser light $\left(2 \mathrm{~W} / \mathrm{cm}^{2}\right)$ for $10 \mathrm{~min}$. The left tumors without laser treatment were used as the control group. The tumor sizes were monitored by measuring the tumor diameters with a caliper. The tumor volume was determined according to the following equation:

$$
\mathrm{V}=\left(\mathrm{A} \times \mathrm{B}^{2}\right) / 2
$$

where $\mathrm{A}$ and $\mathrm{B}$ are the maximum and minimum diameter of the tumor [58]. The temperature change in the tumor region was monitored by a thermal camera for different groups. To evaluate the therapeutic efficacy of PTT, tumor tissues were harvested and fixed in $4 \%$ formaldehyde solution, embedded in paraffin blocks, sectioned into $5-\mu \mathrm{m}$ slices, and mounted on glass slides. After H\&E staining, the sections were observed by using an optical microscope (20× objectives).

\subsection{Degradation test}

Degradation of N-CNDs was investigated by using UV/Vis absorbance. A 1-mL aliquot of N-CNDs ( $2 \mathrm{mg} / \mathrm{mL}$ in PBS, $\mathrm{pH}=7.4$ ) was added to $1 \mathrm{~mL}$ of $\mathrm{H}_{2} \mathrm{O}_{2}$ solution (200 $\mathrm{mM}$ or $2 \mathrm{M}$ ) or serum and incubated at $37^{\circ} \mathrm{C}$. The aliquot was collected at each time point and kept in the deep freezer. The absorption was measured at $450 \mathrm{~nm}(\mathrm{n}=3)$.

\subsection{Toxicity test in vivo}

Eight white Balb/c mice were intravenously injected with $100 \mu \mathrm{L}$ of $20 \mathrm{mg} / \mathrm{mL} \mathrm{N}-\mathrm{CNDs}$ (a dose of $100 \mathrm{mg} / \mathrm{kg}$ ). The other four mice were used as the controls. The mice were sacrificed to collect the major organs and blood for histological and blood analyses at 7 and 28 days post-injection of N-CNDs. Major organs (heart, lung, liver, kidney, spleen) were fixed in $10 \%$ formalin, embedded in paraffin, sectioned, and stained with H\&E. The serum chemistry and complete blood panel were measured at the Center for Evaluation of Biomaterials.

\section{Results}

\subsection{Synthesis of N-CNDs and effect of N-doping}

$\mathrm{N}-\mathrm{CNDs}$ were synthesized by performing solvothermal carbonization of citric acid in the presence of nitric acid as an $\mathrm{N}$ source (Figure 1a). Briefly, an aqueous solution of citric acid with nitric acid was heated at $250^{\circ} \mathrm{C}$ to be initially dehydrated to form polymer-like intermediates. These intermediates were then carbonized to form carbogenic cores, and simultaneously, oleylamine was grafted onto the surface of the core through amidation to suppress undesired inter-particle agglomeration during carbonization. Grafted oleylamine was subsequently replaced by ethanolamine to endow our N-CNDs with water solubility (Figure S2). We controlled the N content of N-CNDs by varying the molarities of nitric acid solutions, and N-CNDs prepared with $0,1,3,5,7$, 10, 12, and $14 \mathrm{M}$ (conc.) nitric acid solutions were coded as N0-, N1-, N3-, N5-, N7-, N10-, N12-, and N14-CNDs, respectively. Transmission electron microscopy showed that the N-CNDs were well dispersed with a particle size of $\sim 3 \mathrm{~nm}$ (Figure 1b). Previously, nanostructures in this size range have been shown to be readily excreted via renal clearance [59]. Figure 1c shows partial graphitic structures or graphitic "clusters" (a few angstroms in size) that were found in the core of N-CNDs after low-temperature $\left(\sim 300^{\circ} \mathrm{C}\right)$ carbonization. These graphitic clusters provide $\Pi$ electrons that would contribute to the formation of the highest occupied molecular orbital (HOMO) and lowest unoccupied molecular orbital (LUMO) levels. X-ray photoelectron spectroscopy data showed that our N-CNDs contained a significant amount of $\mathrm{N}$ atoms that primarily originated from nitric acid and partially surface amide groups formed by ethanolamine, and their $\mathrm{N}$ content varied with the molar concentration of nitric acid (Figure S3). The $\mathrm{N}$ atoms that had lone pairs of electrons (or non-bonding orbitals) generated electronic states in between the HOMO and LUMO 
levels or the so-called intra-gap states, such as non-bonding (n) or defective states. Because intra-gap states reduce the energy gap, as illustrated in Figure $1 d$. N-CNDs that have more $\mathrm{N}$ atoms can generally absorb more photons of lower energy; however, the absorbance values of N-CNDs were saturated at the $\mathrm{N}$ content higher than N7 (Figure 1e). Consistently, photoluminescence spectroscopy of N-CNDs shows that a secondary peak is developed at around $500 \mathrm{~nm}$ as the $\mathrm{N}$ content increases, which also strongly suggests the formation of the N-induced intra-gap states (Figure S4). These intra-gap states have a major role in generating phonons (heat) through Shockley-Read-Hall (SRH) electron-hole recombination or other defect-related paths. Figure $1 f$ indicates the PA spectrum at different nitrogen atoms. N7-CNDs showed the highest PA signal amplitude, which shows a trend similar to that of light absorption. Generally, PA signal amplitude is linearly proportional to absorbance, as shown in Equation 1.

$$
P=\Gamma \cdot \mu_{a} \cdot F
$$

Where $\Gamma$ indicates the Gruneisen parameter, $\mu_{a}$ indicates the optical absorption coefficient, and $F$ indicates the laser fluence. Figure S5 shows that
$\mathrm{N}-\mathrm{CNDs}$ with more $\mathrm{N}$ atoms raised the surrounding temperature faster under NIR $(\lambda=680-808 \mathrm{~nm})$ laser excitation. For instance, under $\lambda=808 \mathrm{~nm}$ excitation, N7-CNDs raised temperatures 20 times faster than did N0-CNDs and four times faster than did N1-CND. The temperature is raised faster because more nitrogen atoms generate more intra-gap states to facilitate carrier trapping from the LUMO to an intra-gap state and carrier relaxation between intra-gap states. We also detected photons of nearly half-gap energy $(\lambda=900-1200 \mathrm{~nm})$ that were emitted in the final stage of SRH recombination (Figure S6), which directly proves the existence of intra-gap states in our N-CNDs. Because the quantum yield $(\Phi)$ of $\mathrm{N}-\mathrm{CNDs}$ in the range of $900-1500 \mathrm{~nm}$ under $808-\mathrm{nm}$ laser excitation was $\sim 1 \%$ (Figure S6), it could be calculated according to Equation 2, which shows that $99 \%$ of excited-state electrons were thermally dissipated through SRH recombination:

$$
\Phi=\frac{\mathrm{k}_{\mathrm{r}}}{\mathrm{k}_{\mathrm{r}}+\mathrm{k}_{\mathrm{nr}}}
$$

where $\mathrm{k}_{\mathrm{r}}$ indicates the radiative decay rate and $\mathrm{k}_{\mathrm{nr}}$ indicates the non-radiative decay rate. a

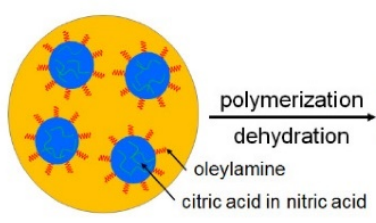

b

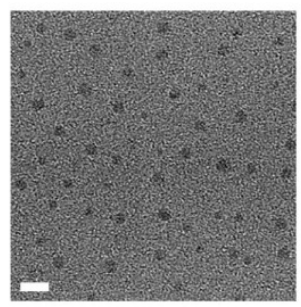

C

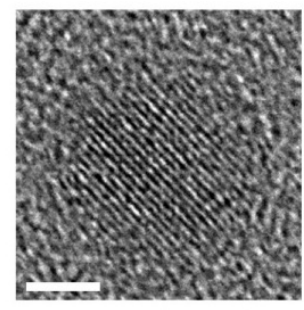

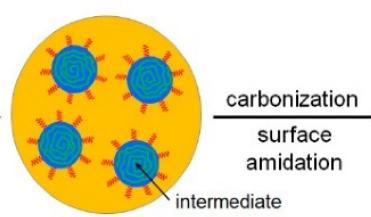

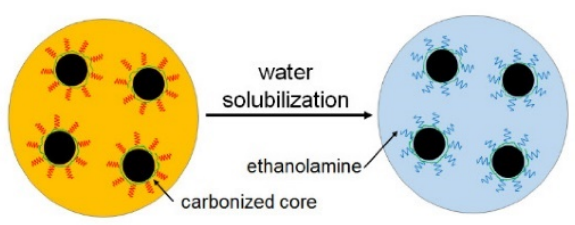

d

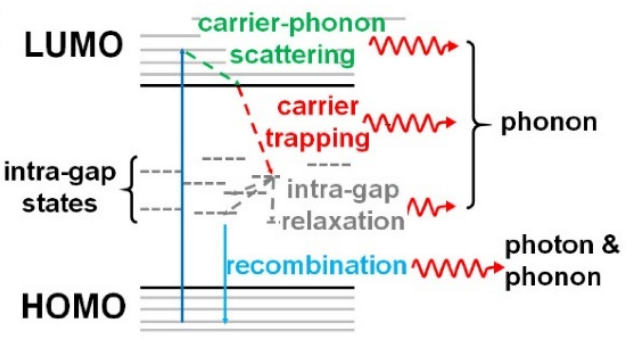

e

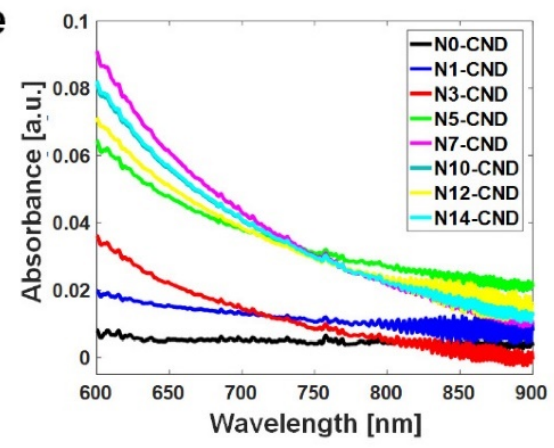

f

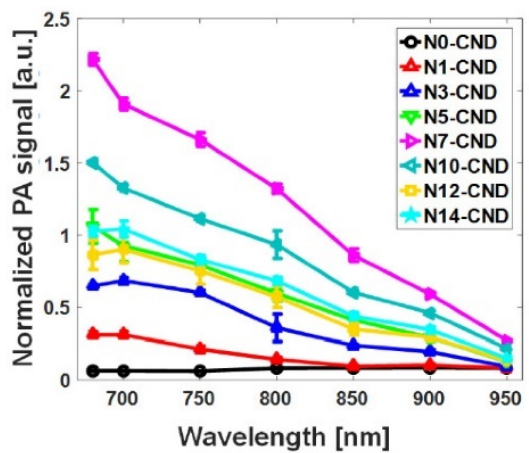

Figure 1. Synthesis of N-CNDs and effect of nitrogen doping. (a) Synthesis of N-CNDs. (b) TEM image of N-CNDs (scale bar = 5 nm). (c) Partial graphitic structures in the core of N-CNDs (scale bar $=2 \mathrm{~nm}$ ). (d) Illustration of electronic structures and nitrogen-induced intra-gap states of N-CNDs. (e) Optical absorption spectrum of N-CNDs as a function of nitric acid concentration. (f) PA amplitude spectrum with different N-doped CNDs. HOMO, highest occupied molecular orbital; LUMO, lowest unoccupied molecular orbital. 


\section{a}

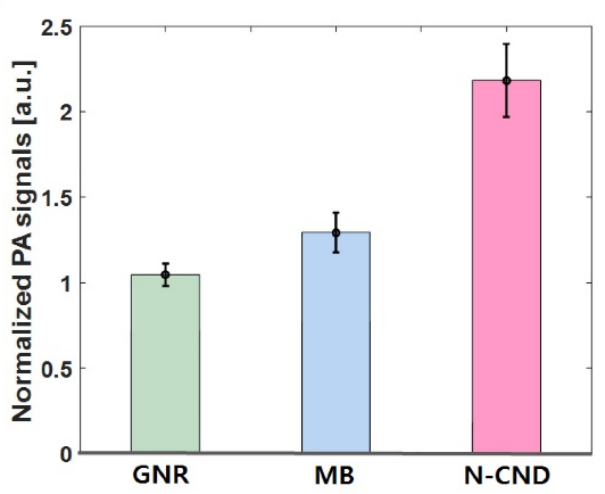

C

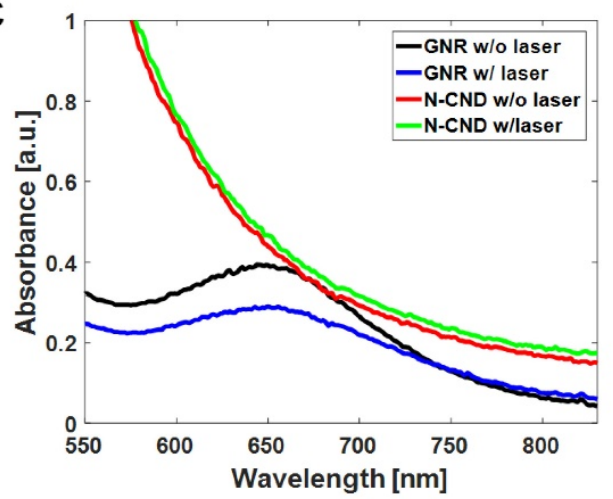

b

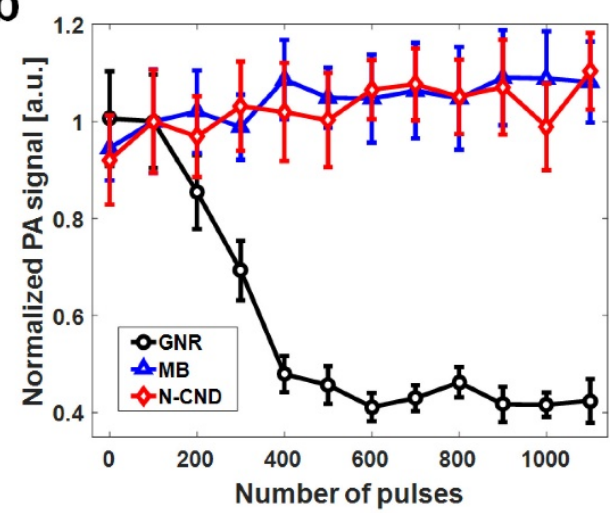

d

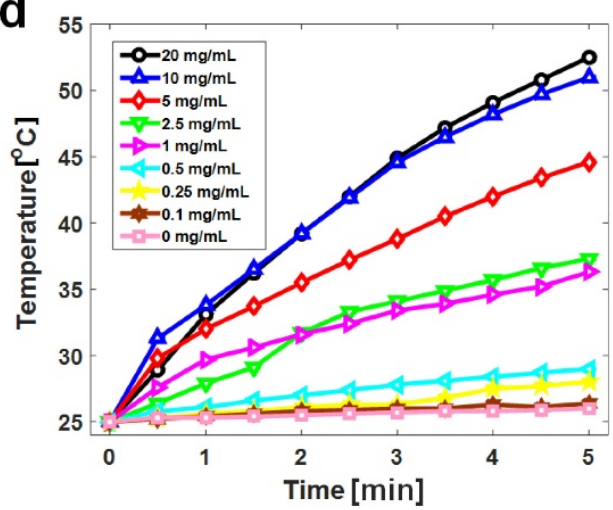

Figure 2. Photoacoustic (PA) and photothermal characteristics of N-CNDs. (a) PA signal amplitude comparison of GNR, MB, and N-CNDs at the same optical density. (b) The stability of the PA responses of GNR, MB, and N-CNDs depending on the number of pulses of laser irradiation. (c) Optical absorption measurement of GNR and N-CNDs with and without laser irradiation. (d) Temperature elevation curves of $\mathrm{N}$-CNDs at different concentrations $(0,0.1,0.25,0.5,1$, $2.5,5,10,20 \mathrm{mg} / \mathrm{mL})$. GNR, gold nanorod; MB, methylene blue.

\subsection{PA and photothermal characteristics of N-CNDs}

The optical absorbance of N-CNDs increased with increasing concentration (Figure S7a), which indicated that N-CNDs have broad absorption in the NIR region (680-800 $\mathrm{nm}$ ) and were well matched to the PA amplitude spectral distribution at the same concentration (Figure S7b). Although PA signal amplitude decreased with increasing wavelength, the PA signal could still be detected at $900 \mathrm{~nm}$. The PA sensitivity at $680 \mathrm{~nm}$ was measured by testing the PA signal amplitude of N-CNDs at different concentrations; this measurement showed a linear relationship. The detection limit of the N-CNDs was 1 $\mathrm{mg} / \mathrm{mL}$ (Figure S8). To investigate the performance of $\mathrm{N}-\mathrm{CNDs}$ as a contrast agent, we directly compared the PA signals with those of popular conventional PA contrast agents, such as GNR and MB (Figure S9). At the same optical density, the normalized PA signal amplitude of N-CNDs $(2.2 \pm 0.2)$ was approximately two times higher than those of GNR $(1.0 \pm 0.1)$ and MB $(1.3 \pm 0.1)$ (Figure 2a). This result implies that $\mathrm{N}-\mathrm{CNDs}$ have higher heat conversion efficiency than those of GNR and MB at the same optical density, which contains strong nonradiative energy decay, high heat conductance and capacity, and weak optical scattering [19, 60]. In addition, N-CNDs showed strong stability of the PA response to high-energy pulsed laser irradiation (Figure 2b). Pulsed laser at a 680-nm optical wavelength $\left(15 \mathrm{~mJ} / \mathrm{cm}^{2}\right)$ was projected onto the samples. The PA signal amplitudes of $\mathrm{N}-\mathrm{CNDs}$ and $\mathrm{MB}$ were relatively stable, but the PA signals from GNR decreased by $50 \%$ during the 300 pulses and entered the photobleaching state. Furthermore, the optical stability of N-CNDs for PTT was assessed by comparing GNR (Figure 2c). The optical absorption of N-CNDs varied from 0.43 to 0.40 , whereas GNR varied from 0.39 to 0.28 under $660-\mathrm{nm}$ continuous wave laser excitation over $10 \mathrm{~min}$. To examine the photothermal properties of CNDs, the temperature changes of an aqueous solution of CNDs at diverse concentrations $(0.1-20 \mathrm{mg} / \mathrm{mL})$ were measured under irradiation by a 808 -nm NIR laser ( 2 $\mathrm{W} / \mathrm{cm}^{2}$ ), and DI water was used as the control (Figure $2 \mathrm{~d})$.

\subsection{In vivo/ex vivo PA sentinel lymph node mapping}

SLNs and photoacoustically vascular networks were acoustic-resolution reflection-mode PA imaging 
system at $680-\mathrm{nm}$ optical wavelength (Figure S1). Detecting SLNs is a clinically important issue for the diagnosis of metastatic cancer [13]. Figures 3a-c show the PA maximum amplitude projection (MAP) images, which clearly indicate the position of $\mathrm{N}-\mathrm{CNDs}$ draining from an SLN. Figures $3 \mathrm{e}-\mathrm{g}$ show the depth-resolved cross-sectional PA images of the corresponding yellow line in Figures $3 a-c$, respectively. Additionally, the depth information of the PA MAP image (Figure 3b) was encoded by a pseudo color, as shown in Figure 3d. Normally, the SLNs are located $\sim 3 \mathrm{~mm}$ below the skin surface. Before injecting N-CNDs, the PA MAP image did not delineate the SLN but only showed the vasculature. After intradermal injection of N-CNDs, the PA signal amplitude of the SLN suddenly increased at 30 mins (167.2 $\pm 21.9 \%)$; subsequently, it decreased continuously until $180 \mathrm{~min}(33.1 \pm 9.9 \%)(\mathrm{n}=3$, Figure $3 \mathrm{~h})$. These results indicate that N-CNDs were initially collected and then eliminated from the SLN after circulating in the lymphatic system. To validate the results of the in vivo PA imaging, ex vivo PA imaging was performed by excising lymph nodes containing $\mathrm{N}-\mathrm{CNDs}$ and normal lymph nodes from the axial region of rats (Figure 3i). Only the excised lymph nodes containing N-CNDs were detected in the PA MAP images (Figure 3j), whereas normal ones did not appear photoacoustically. Normally, the highest PA signal was measured from the SLN. However, an SLN injected with N-CNDs did not show the highest PA signal because the N-CNDs degraded. As the $\mathrm{N}-\mathrm{CNDs}$ were fading away from the SLN, the strongest PA signal was observed at L2 (second lymph node). The PA contrast of the SLN and L2 were determined to be $299 \pm 86 \%$ and $512 \pm 65 \%$, respectively (Figure $3 \mathrm{k}$ ). Therefore, these results demonstrate the potential usefulness of N-CNDs for PA lymphoscintigraphy in clinical axillary staging of cancer.
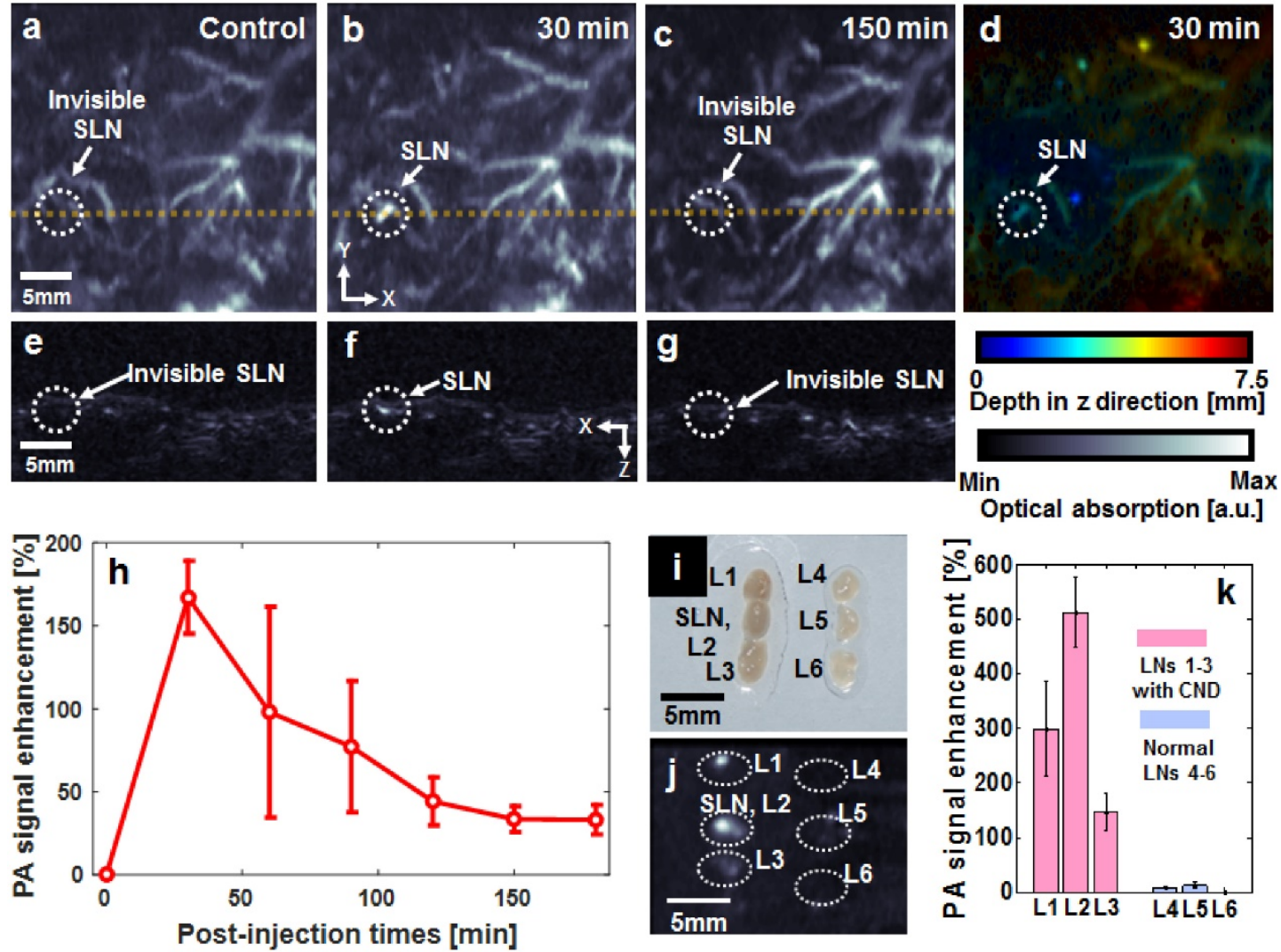

Figure 3. In vivo photoacoustic (PA) sentinel lymph node (SLN) mapping with N-CNDs injection. (a) PA maximum amplitude projection (MAP) image before N-CNDs injection. (b) PA MAP image 30 min after N-CNDs injection. (c) PA MAP image after 150 min of N-CNDs injection. (d) Depth-encoded PA MAP image after 30 min of N-CNDs injection. (e-g) Corresponding PA B-mode images of the dashed line area in (a-c). (h) PA signal profile in the SLN region from before injection to 180 min after the N-CNDs injection. (i) and (j) Photograph and PA MAP image, respectively, of SLNs excised after the N-CNDs injection (L1-L3) and control (L4-L6). (k) PA signal of the excised SLNs. SLN, sentinel lymph node; L, lymph node. 

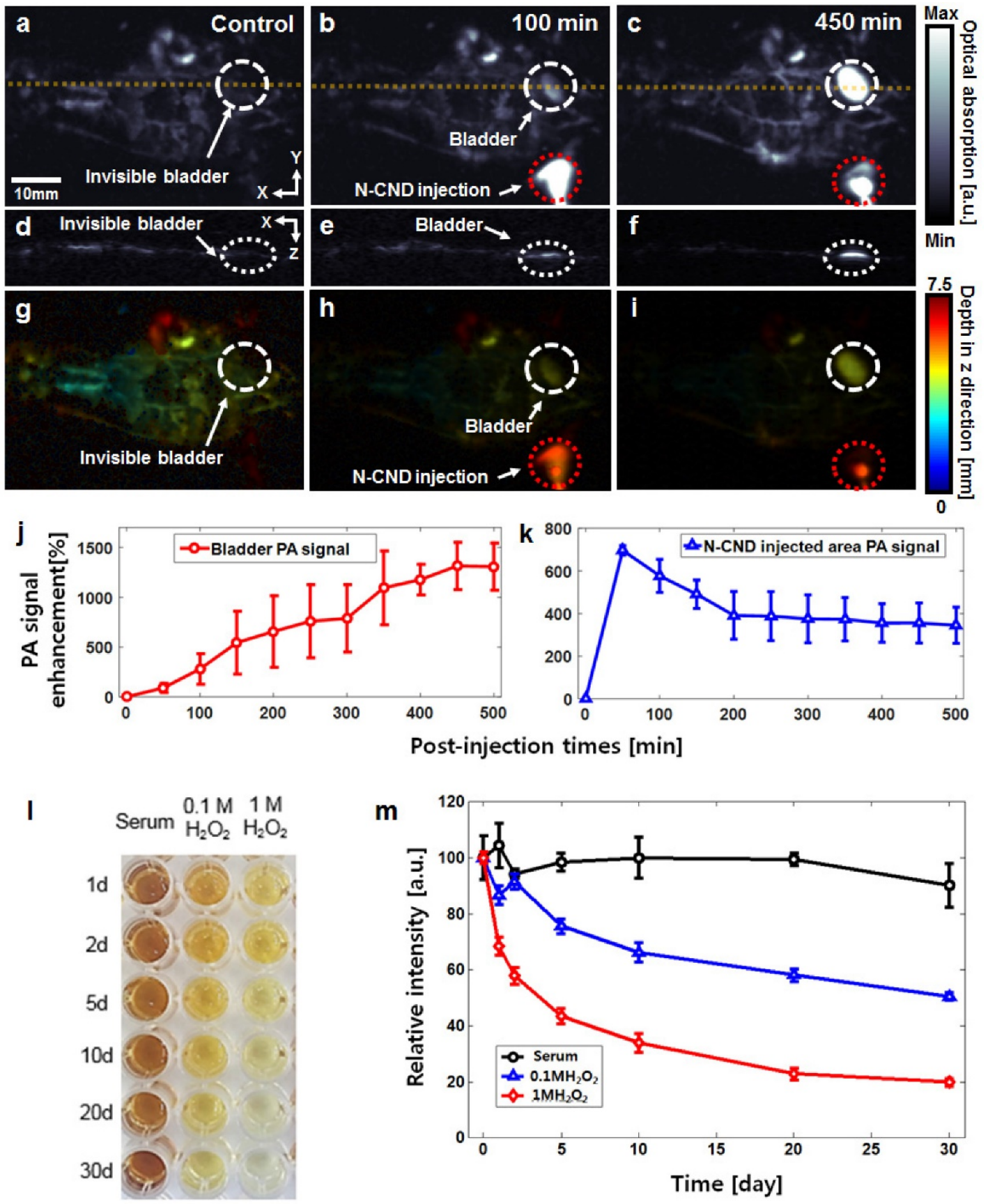

Figure 4. In vivo photoacoustic (PA) bladder imaging and degradation test using N-CNDs. (a) Coronal (front) PA maximum amplitude projection (MAP) image before N-CNDs injection. (b) and (c) Coronal PA MAP image after $100 \mathrm{~min}$ and $450 \mathrm{~min}$ of N-CNDs injection. (d-f) Corresponding PA B-mode images at the dashed line region of $(\mathrm{a}-\mathrm{c})$. $(\mathrm{g}-\mathrm{i})$ Corresponding depth-encoded PA MAP images of the PA MAP images of $(\mathrm{a}-\mathrm{c})$. (j) and (k) PA signal profile in the bladder region (white dashed circle in $(\mathrm{a}-\mathrm{c})$ ) and injected area (red dashed circle in $(\mathrm{a}-\mathrm{c})$ ) from before injection to $500 \mathrm{~min}$ after the N-CNDs injection. (I) and (m) Serum biochemistry assay of N-CNDs-injected mice.

\subsection{In vivo whole-body PA imaging of renal clearance of N-CNDs}

After subcutaneous injection of N-CNDs, whole-body PA imaging was performed to determine the bodily distribution and clearance of N-CNDs. Figures $4 \mathrm{a}-\mathrm{c}$ and Figures $4 \mathrm{~d}-\mathrm{f}$ present the whole-body PA MAP images and depth-resolved cross-sectional PA images of the yellow line, respectively. Although the blood vessels are clearly shown in the control PA images (Figs. 4a and d), the bladder was invisible because of its transparency. Figures $4 \mathrm{~g}-\mathrm{i}$ show the depth-coded PA MAP images corresponding to Figures $4 a-c$, respectively. At 100 min post-injection of $\mathrm{N}-\mathrm{CNDs}$, an increased PA signal appeared in the bladder (Figs. $4 \mathrm{~b}$ and $4 \mathrm{e}$ ). Over time, the PA signal amplitude of the bladder was generally enhanced up to $450 \mathrm{~min}(1314 \pm 237 \%)(\mathrm{n}=3$, Figure $4 \mathrm{j})$. However, the PA signal amplitude of the injection area 
decreased up to $500 \min (124 \pm 55 \%)(\mathrm{n}=3$, Figure $4 \mathrm{k})$. These PA experimental results revealed that $\mathrm{N}-\mathrm{CNDs}$ could be removed from the body by renal clearance after subcutaneous injection. The results showed that $\mathrm{N}-\mathrm{CNDs}$ can contribute to the diagnosis of urinary tract diseases, including leaks and fistulas [61].

\subsection{Degradation and in vivo toxicity}

$\mathrm{N}-\mathrm{CNDs}$ degradation was investigated using $\mathrm{UV} / \mathrm{Vis}$ absorbance (Figs. $4 \mathrm{l}$ and $\mathrm{m}$ ). Upon exposure to $\mathrm{H}_{2} \mathrm{O}_{2}$, the formation rate of hydroxyl radials increased in two ways: reduction of $\mathrm{H}_{2} \mathrm{O}_{2}$ at the conduction band and self-decomposition by irradiation. The former caused degradation of $\mathrm{N}$-CNDs. As seen in Figure 4l, the decolorization rate increased when the $\mathrm{H}_{2} \mathrm{O}_{2}$ concentration increased from 0 to $1 \mathrm{M}$. The toxicity of N-CNDs was investigated in vivo. We monitored the behaviors of mice after intravenous injection of N-CNDs (100 $\mathrm{mg} / \mathrm{kg}$ ), and no apparent symptoms of toxic effects were observed within 1 month. The mice were sacrificed at days 7 and 30 for careful necropsy, and PBS treated mice were used as controls. The mice hearts, lungs, livers, kidneys, and spleens were fixed, sliced, and stained by hematoxylin and eosin (H\&E) (Figure S10). Because of the high renal clearance rate of N-CNDs in the body, no noticeable organ destruction or inflammatory lesions were observed in the hearts, lungs, livers, kidneys, and spleens of the mice at 7 and 30 days, similar to the control group results. To further investigate the potential toxicology of N-CNDs in the body, a complete blood panel test and serum biochemistry assay were performed on $\mathrm{N}$-CNDs-injected $(100 \mathrm{mg} / \mathrm{kg})$ white Balb/c mice at days 7 and 30. The measured parameter results were within normal ranges (Figure S11). The toxicity test results indicated that $\mathrm{N}-\mathrm{CNDs}$ were biocompatible in vivo in mice for at least 30 days at our tested dose.

\subsection{In vitrolin vivo PTT using N-CNDs}

We investigated the in vitro cytotoxicity of $\mathrm{N}-\mathrm{CNDs}$ and PTT efficacy in FL83B and HepG2. A high concentration $(10 \mathrm{mg} / \mathrm{mL})$ of N-CNDs caused no cytotoxicity in FL83B cells or HepG2 cells without laser irradiation (Figure S12a). In contrast, with 808-nm NIR laser irradiation, the viabilities of HepG2 cells decreased significantly as the concentration of $\mathrm{N}-\mathrm{CNDs}$ increased (Figure S12b). Subsequently, a live/dead assay was performed to further verify the PTT efficacy of N-CNDs. Figure S12c shows that the cells incubated with N-CNDs and exposed to 808-nm laser light $\left(2 \mathrm{~W} / \mathrm{cm}^{2}, 10 \mathrm{~min}\right)$ exhibited red fluorescence, which indicated cancer cell death. Figure S12d shows confocal images of FL83B cells and
HepG2 cells treated with N-CNDs at excitation wavelengths of 405, 488, 561, and $633 \mathrm{~nm}$. In the control group, all cells lived, as indicated by the green fluorescence. Based on the in vitro results, we further investigated the in vivo PTT of cancers using N-CNDs. Balb/c nude xenograft HepG2 tumor model mice (five mice per group) with a tumor volume of $27 \mathrm{~mm}^{3}$ were intratumorally injected with $\mathrm{N}-\mathrm{CNDs}$ solution $(20 \mathrm{mg} / \mathrm{mL}, 100 \mu \mathrm{L}$ ) and PBS as a control. The tumor regions of the PBS + laser group and N-CNDs + laser group were irradiated with the laser for $10 \mathrm{~min}$, whereas the laser-untreated group was not irradiated for comparison. The temperature of the tumor area was monitored by a thermal camera at specific time points (Figures $5 a$ and $b$ ). Under NIR laser irradiation, the tumor surface temperature of the N-CNDs + laser group rapidly increased from $\sim 27^{\circ} \mathrm{C}$ to $50^{\circ} \mathrm{C}$. The temperature change causes hyperthermia that can kill cancer cells. In contrast, the temperature of the tumor region of the control group and non-irradiated surrounding normal tissues showed no temperature change. To confirm the PTT efficacy of the N-CNDs, we monitored the tumor growth rates. After treatment with PBS regardless of laser irradiation, the tumor only continued to grow. Also, the tumor treated with N-CNDs without laser irradiation, the tumor volume increased over time. However, the tumor that was treated with N-CNDs and 808-nm NIR laser irradiation together completely disappeared (Figs. 5c and d). The anti-tumor effect was assessed by histological analysis of tumor tissues by H\&E staining and TUNEL assay 12 hours after laser irradiation. The tumor tissues treated with N-CNDs + laser irradiation showed extensive nuclear shrinkage and fragmentation, which suggested that the PTT was enhanced in vivo. In contrast, cell destruction was negligible in tumor tissues treated with PBS, PBS + laser irradiation, and N-CNDs. From the fluorescence TUNEL assay (Figure 5e), the tumor tissues treated with N-CNDs + laser irradiation showed green fluorescence, which indicated cellular apoptosis. Normal areas stained blue, and TUNEL-positive areas stained green. In contrast, the tumor sections treated with PBS, N-CNDs, and PBS + laser irradiation showed only blue fluorescence. These results confirmed the feasibility of N-CNDs for PTT of cancer.

\section{Conclusion}

We successfully verified the feasibility of a biocompatible optical-absorbing material, N-doped CND, from PA imaging of SLN and bladder, and cancer ablation PTT. The relative PA signal increased in SLNs after injection of N-CNDs, and degraded after circulating through the lymphatic system. 
Additionally, the PA signal in the bladder region increased dramatically after injection of N-CNDs, which indicated that N-CNDs were eliminated effectively in the urine. In cancer ablation PTT, the $\mathrm{N}$-CNDs-injected region after laser irradiation treatment showed complete tumor ablation without recurrence. The light-absorbing material, N-CNDs, can be a promising agent for enhancement of optical contrast in PA imaging and to enhance the effect of PTT. a

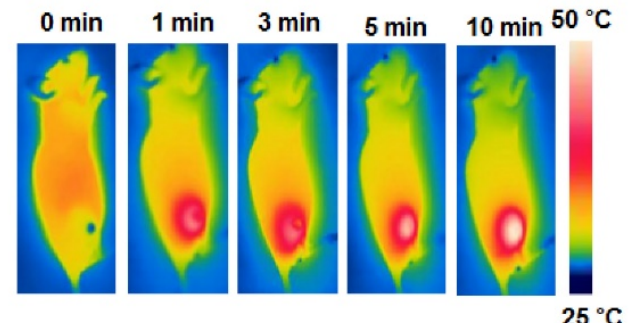

C

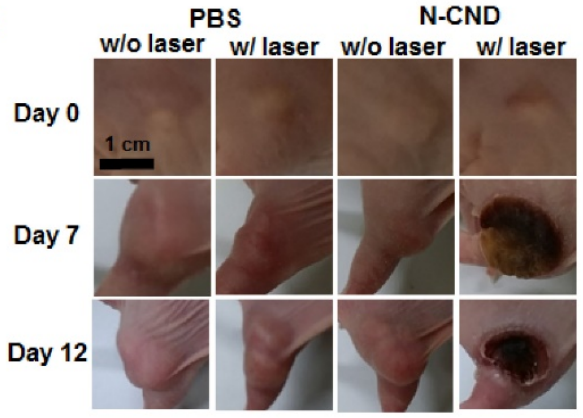

e
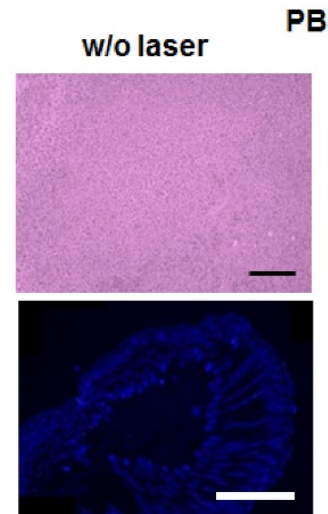

BS

wl laser
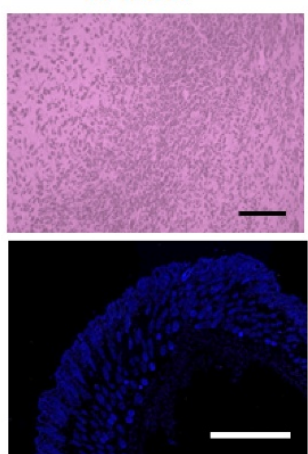

b

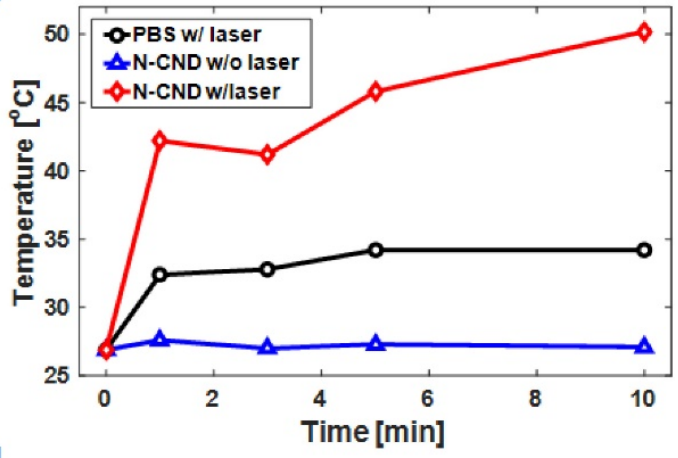

d

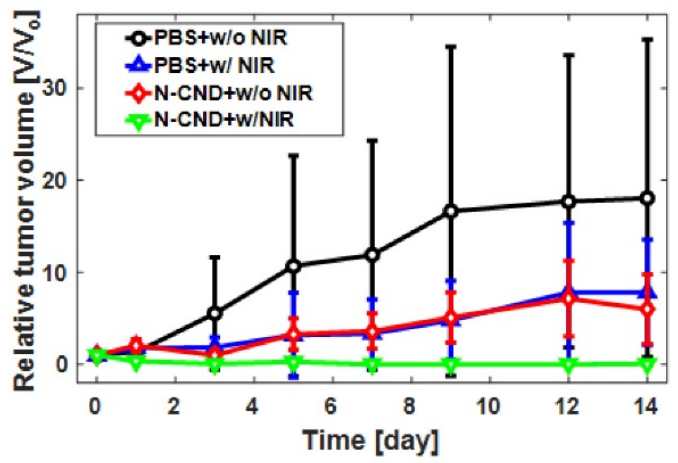

N-CND

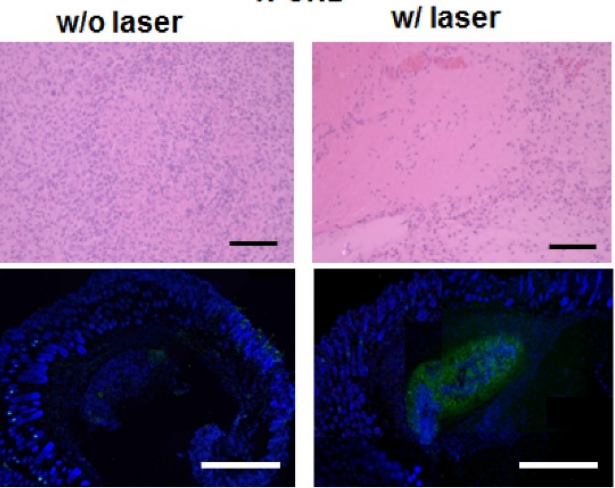

Figure 5. Photothermal therapy (PTT) using N-CNDs (a) Thermal camera images of N-CNDs injection with NIR laser irradiation. (b) Temperature profile of the injected area with and without NIR laser irradiation. (c) and (d) PTT monitoring and tumor volume profile of PBS and N-CNDs-injected models. (e) Histological analysis of tumor tissues with H\&E staining and TUNEL assay 12 hours after PBS and N-CNDs injection without and with NIR laser irradiation. (black scale bar $=100$ $\mu \mathrm{m}$, white scale bar $=1000 \mu \mathrm{m})$.

\section{Supplementary Material}

Supplementary figures.

http://www.thno.org/v06p2196s1.pdf

\section{Abbreviations}

N, Nitrogen; CNDs, Carbon nanodots; N-CNDs, Nitrogen-doped carbon nanodots; PA, Photoacoustic; PTT, photothermal therapy; SLNs, sentinel lymph nodes; NIR, near-infrared; GNR, gold nanorod; MB, methylene blue; DMEM, Dulbecco's modified Eagle's medium; FBS, fetal bovine serum; PBS, Phosphate-buffered solution; MTT, diphenyl-2Htetrazolium bromide; PI, propidium iodide; HOMO, Highest occupied molecular orbital; LUMO, Lowest unoccupied molecular orbital; SRH, Shockley-ReadHall; MAP, Maximum amplitude projection. 


\section{Acknowledgments}

Changho Lee, Woosung Kwon, and Songeun Beack contributed equally to this work. We thank Hyun-Jin Park (National Institute for Nanomaterials Technology) for providing TEM images. This work was supported by the research funds from an IITP ICT Consilience Creative Program (IITP-2015-R0346-151007), an NRF Engineering Research Center grant (NRF-2011-0030075), Basic Science Program (NRF-2016R1C1B1011830), a Bio \& Medical Technology Development Program (2015R1A2A1A15053779) of the Ministry of Science, ICT, Future Planning, a grant (KHIDI-HI15C1817), the Korean Health Technology R\&D Project (HI114C1658) from the Ministry of Health \& Welfare, the Marine Biotechnology Program (20150220) funded by the Ministry of Oceans and Fisheries, Sookmyung Women's University Research Grant (1-1603-2001), and the Samsung Research Funding Center of Samsung Electronics (SRFC-MA1401-07).

\section{Competing Interests}

The authors have declared that no competing interest exists.

\section{References}

1. Sanvicens N, Marco MP. Multifunctional nanoparticles-properties and prospects for their use in human medicine. Trends Biotechnol. 2008; 26: 425-33.

2. Chen J, Yang M, Zhang Q, Cho EC, Cobley CM, Kim C, et al. Gold nanocages: a novel class of multifunctional nanomaterials for theranostic applications. Adv Funct Mater. 2010; 20: 3684-94.

3. Jin Y, Jia C, Huang S-W, O'Donnell M, Gao X. Multifunctional nanoparticles as coupled contrast agents. Nat commun. 2010; 1: 41.

4. Lee D-E, Koo H, Sun I-C, Ryu JH, Kim K, Kwon IC. Multifunctional nanoparticles for multimodal imaging and theragnosis. Chem Soc Rev. 2012; 41: 2656-72

5. Roy I, Shetty D, Hota R, Baek K, Kim J, Kim C, et al. A Multifunctional Subphthalocyanine Nanosphere for Targeting, Labeling, and Killing of Antibiotic-Resistant Bacteria. Angew Chem. 2015; 127: 15367-70.

6. Pissuwan D, Valenzuela SM, Cortie MB. Therapeutic possibilities of plasmonically heated gold nanoparticles. Trends Biotechnol. 2006; 24: 62-7

7. Wang C, Cheng L, Liu Z. Drug delivery with upconversion nanoparticles for multi-functional targeted cancer cell imaging and therapy. Biomaterials. 2011; 32: 1110-20.

8. Kim C, Favazza C, Wang LV. In Vivo Photoacoustic Tomography of Chemicals: High-Resolution Functional and Molecular Optical Imaging at New Depths. Chem Rev. 2010; 110: 2756-82.

9. Wang LV, Hu S. Photoacoustic Tomography: In Vivo Imaging from Organelles to Organs. Science. 2012; 335: 1458-62.

10. Kim J, Park S, Lee C, Kim JY, Kim C. Organic Nanostructures for Photoacoustic Imaging. ChemNanoMat. 2016; 2: 156-66.

11. Jeon M, Kim J, Kim C. Multiplane spectroscopic whole-body photoacoustic imaging of small animals in vivo. Med Biol Eng Comput. 2016; 54: 283-94.

12. Jeon M, Song W, Huynh E, Kim J, Kim J, Helfield BL, et al. Methylene blue microbubbles as a model dual-modality contrast agent for ultrasound and activatable photoacoustic imaging. J biomed opt. 2014; 19: 016005.

13. Lee C, Kim J, Zhang Y, Jeon M, Liu C, Song L, et al. Dual-color photoacoustic lymph node imaging using nanoformulated naphthalocyanines. Biomaterials. 2015; 73: 142-8.

14. Lee M-Y, Lee C, Jung HS, Jeon M, Kim KS, Yun SH, et al. Biodegradable Photonic Melanoidin for Theranostic Applications. ACS Nano. 2015; 10: 822-31.

15. Liu X, Lee C, Law W-C, Zhu D, Liu M, Jeon M, et al. Au-Cu2-x Se Heterodimer Nanoparticles with Broad Localized Surface Plasmon Resonance as Contrast Agents for Deep Tissue Imaging. Nano Lett. 2013; 13: 4333-9.

16. Liu Y, Kang N, Lv J, Zhou Z, Zhao Q, Ma L, et al. Deep Photoacoustic/Luminescence/Magnetic Resonance Multimodal Imaging in Living Subjects Using High-Efficiency Upconversion Nanocomposites. Adv Mater. 2016.
17. Liu Y, Nie L, Chen X. Photoacoustic Molecular Imaging: From Multiscale Biomedical Applications Towards Early-Stage Theranostics. Trends Biotechnol. 2016; 34: 420-33

18. Yao J, Maslov KI, Zhang Y, Xia Y, Wang LV. Label-free oxygen-metabolic photoacoustic microscopy in vivo. J biomed opt. 2011; 16: 076003--11.

19. Zhang Y, Jeon M, Rich LJ, Hong H, Geng J, Zhang Y, et al. Non-invasive multimodal functional imaging of the intestine with frozen micellar naphthalocyanines. Nat Nanotechnol. 2014; 9: 631-8.

20. Liu J, Zheng X, Yan L, Zhou L, Tian G, Yin W, et al. Bismuth Sulfide Nanorods as a Precision Nanomedicine for in Vivo Multimodal Imaging-Guided Photothermal Therapy of Tumor. ACS Nano. 2015; 9: 696-707.

21. Huang P, Lin J, Li W, Rong P, Wang Z, Wang S, et al. Biodegradable Gold Nanovesicles with an Ultrastrong Plasmonic Coupling Effect for Photoacoustic Imaging and Photothermal Therapy. Angew Chem Int Edit. 2013; 52: 13958-64.

22. Cheng L, Yang K, Chen Q, Liu Z. Organic Stealth Nanoparticles for Highly Effective in Vivo Near-Infrared Photothermal Therapy of Cancer. ACS Nano. 2012; 6: 5605-13.

23. Yang K, Zhang S, Zhang G, Sun X, Lee S-T, Liu Z. Graphene in Mice: Ultrahigh In Vivo Tumor Uptake and Efficient Photothermal Therapy. Nano Lett. 2010; 10: 3318-23.

24. Nikoobakht B, El-Sayed MA. Preparation and growth mechanism of gold nanorods (NRs) using seed-mediated growth method. Chem Mater. 2003; 15 : 1957-62.

25. Kim J-W, Galanzha EI, Shashkov EV, Moon H-M, Zharov VP. Golden carbon nanotubes as multimodal photoacoustic and photothermal high-contrast molecular agents. Nat Nanotechnol. 2009; 4: 688-94.

26. Bardhan R, Lal S, Joshi A, Halas NJ. Theranostic nanoshells: from probe design to imaging and treatment of cancer. Accounts Chem Res. 2011; 44: 936-46.

27. Liu X, Law WC, Jeon M, Wang X, Liu M, Kim C, et al. Cu2-xSe nanocrystals with localized surface plasmon resonance as sensitive contrast agents for in vivo photoacoustic imaging: Demonstration of sentinel lymph node mapping. Adv Healthc Mater. 2013; 2: 952-7.

28. Yang H-W, Liu H-L, Li M-L, Hsi I-W, Fan C-T, Huang C-Y, et al. Magnetic gold-nanorod/PNIPAAmMA nanoparticles for dual magnetic resonance and photoacoustic imaging and targeted photothermal therapy. Biomaterials. 2013; 34: 5651-60.

29. Nie L, Wang S, Wang X, Rong P, Ma Y, Liu G, et al. In vivo volumetric photoacoustic molecular angiography and therapeutic monitoring with targeted plasmonic nanostars. Small. 2014; 10: 1585-93.

30. Koo J, Jeon M, Oh Y, Kang HW, Kim J, Kim C, et al. In vivo non-ionizing photoacoustic mapping of sentinel lymph nodes and bladders with ICG-enhanced carbon nanotubes. Phys Med Biol. 2012; 57: 7853.

31. Yang K, Hu L, Ma X, Ye S, Cheng L, Shi X, et al. Multimodal imaging guided photothermal therapy using functionalized graphene nanosheets anchored with magnetic nanoparticles. Adv Mater. 2012; 24: 1868-72.

32. Jin Y, Li Y, Ma X, Zha Z, Shi L, Tian J, et al. Encapsulating tantalum oxide into polypyrrole nanoparticles for X-ray $\mathrm{CT} /$ photoacoustic bimodal imaging-guided photothermal ablation of cancer. Biomaterials. 2014; 35: 5795-804.

33. Liang X, Li Y, Li X, Jing L, Deng Z, Yue X, et al. PEGylated Polypyrrole Nanoparticles Conjugating Gadolinium Chelates for Dual-Modal MRI/Photoacoustic Imaging Guided Photothermal Therapy of Cancer. Adv Funct Mater. 2015; 25: 1451-62.

34. Lyu $\mathrm{Y}$, Fang $\mathrm{Y}$, Miao $\mathrm{O}$, Zhen $\mathrm{X}$, Ding $\mathrm{D}, \mathrm{Pu} \mathrm{K}$. Intraparticle Molecular Orbital Engineering of Semiconducting Polymer Nanoparticles as Amplified Theranostics for in Vivo Photoacoustic Imaging and Photothermal Therapy. ACS Nano. 2016; 10: 4472-81.

35. Rieffel J, Chen F, Kim J, Chen G, Shao W, Shao S, et al. Hexamodal Imaging with Porphyrin-Phospholipid-Coated Upconversion Nanoparticles. Adv Mater. 2015; 27: 1785-90.

36. Lovell JF, Jin CS, Huynh E, Jin H, Kim C, Rubinstein JL, Chan WC, Cao W, Wang LV, Zheng G. Porphysome nanovesicles generated by porphyrin bilayers for use as multimodal biophotonic contrast agents. Nat Mater. 2011; 10: 324-32

37. Chen Y-S, Frey W, Kim S, Homan K, Kruizinga P, Sokolov K, et al. Enhanced thermal stability of silica-coated gold nanorods for photoacoustic imaging and image-guided therapy. Opt express. 2010; 18: 8867-78.

38. Tian Q, Hu J, Zhu Y, Zou R, Chen Z, Yang S, et al. Sub-10 nm Fe3O4@ Cu2-x S Core-Shell Nanoparticles for Dual-Modal Imaging and Photothermal Therapy. J Am Chem Soc. 2013; 135: 8571-7.

39. Gad SC, Sharp KL, Montgomery C, Payne JD, Goodrich GP. Evaluation of the toxicity of intravenous delivery of auroshell particles (gold-silica nanoshells). Int J Toxicol. 2012; 31: 584-94

40. Li Z, Huang P, Zhang X, Lin J, Yang S, Liu B, et al. RGD-conjugated dendrimer-modified gold nanorods for in vivo tumor targeting and photothermal therapy. Mol Pharm. 2009; 7: 94-104.

41. Huang P, Bao L, Zhang C, Lin J, Luo T, Yang D, et al. Folic acid-conjugated silica-modified gold nanorods for X-ray/CT imaging-guided dual-mode radiation and photo-thermal therapy. Biomaterials. 2011; 32: 9796-809.

42. Bhunia SK, Saha A, Maity AR, Ray SC, Jana NR. Carbon Nanoparticle-based Fluorescent Bioimaging Probes. Sci Rep. 2013; 3: 1473.

43. Hong G, Diao S, Antaris AL, Dai H. Carbon Nanomaterials for Biological Imaging and Nanomedicinal Therapy. Chem Rev. 2015; 115: 10816-906. 
44. Cao L, Wang X, Meziani MJ, Lu F, Wang H, Luo PG, et al. Carbon dots for multiphoton bioimaging. J Am Chem Soc. 2007; 129: 11318-9.

45. Li H, Kang Z, Liu Y, Lee S-T. Carbon nanodots: synthesis, properties and applications. J Mater Chem. 2012; 22: 24230-53.

46. Li W, Zhang Z, Kong B, Feng S, Wang J, Wang L, et al. Simple and Green Synthesis of Nitrogen-Doped Photoluminescent Carbonaceous Nanospheres for Bioimaging. Angew Chem Int Edit. 2013; 52: 8151-5.

47. Qu S, Wang X, Lu Q, Liu X, Wang L. A Biocompatible Fluorescent Ink Based on Water-Soluble Luminescent Carbon Nanodots. Angew Chem Int Edit. 2012; 51: 12215-8.

48. Sun Y-P, Zhou B, Lin $Y$, Wang W, Fernando KS, Pathak $P$, et al Quantum-sized carbon dots for bright and colorful photoluminescence. J Am Chem Soc. 2006; 128: 7756-7.

49. Xu Z-Q, Lan J-Y, Jin J-C, Dong P, Jiang F-L, Liu Y. Highly Photoluminescent Nitrogen-Doped Carbon Nanodots and Their Protective Effects against Oxidative Stress on Cells. ACS Appl Mater Inter. 2015; 7: 28346-52.

50. Xu Z-Q, Yang L-Y, Fan X-Y, Jin J-C, Mei J, Peng W, et al. Low temperature synthesis of highly stable phosphate functionalized two color carbon nanodots and their application in cell imaging. Carbon. 2014; 66: 351-60.

51. Yang S-T, Cao L, Luo PG, Lu F, Wang X, Wang H, et al. Carbon dots for optical imaging in vivo. J Am Chem Soc. 2009; 131: 11308-9.

52. Jiang K, Sun S, Zhang L, Lu Y, Wu A, Cai C, et al. Red, Green, and Blue Luminescence by Carbon Dots: Full-Color Emission Tuning and Multicolor Cellular Imaging. Angew Chem Int Edit. 2015; 54: 5360-3.

53. Ge J, Jia Q, Liu W, Guo L, Liu Q, Lan M, et al. Red-Emissive Carbon Dots for Fluorescent, Photoacoustic, and Thermal Theranostics in Living Mice. Adv Mater. 2015; 27: 4169-77.

54. Tang J, Kong B, Wu H, Xu M, Wang Y, Wang Y, et al. Carbon Nanodots Featuring Efficient FRET for Real-Time Monitoring of Drug Delivery and Two-Photon Imaging. Adv Mater. 2013; 25: 6569-74.

55. Tao H, Yang K, Ma Z, Wan J, Zhang Y, Kang Z, et al. In Vivo NIR Fluorescence Imaging, Biodistribution, and Toxicology of Photoluminescent Carbon Dots Produced from Carbon Nanotubes and Graphite. Small. 2012; 8: 281-90.

56. Sahu S, Behera B, Maiti TK, Mohapatra S. Simple one-step synthesis of highly luminescent carbon dots from orange juice: application as excellent bio-imaging agents. Chem Commun. 2012; 48: 8835-7.

57. Huang $\mathrm{P}$, Lin J, Wang $\mathrm{X}$, Wang Z, Zhang $\mathrm{C}$, He $\mathrm{M}$, et al. Light-Triggered Theranostics Based on Photosensitizer-Conjugated Carbon Dots for Simultaneous Enhanced-Fluorescence Imaging and Photodynamic Therapy. Adv Mater. 2012; 24: 5104-10.

58. Hoeck JD, Jandke A, Blake SM, Nye E, Spencer-Dene B, Brandner S, et al. Fbw7 controls neural stem cell differentiation and progenitor apoptosis via Notch and c-Jun. Nat Neurosci. 2010; 13: 1365-72.

59. Soo Choi H, Liu W, Misra P, Tanaka E, Zimmer JP, Itty Ipe B, et al. Renal clearance of quantum dots. Nat Biotechnol. 2007 : 25: 1165-70.

60. $\mathrm{Pu} \mathrm{K}$, Shuhendler AJ, Jokerst JV, Mei J, Gambhir SS, Bao Z, et al. Semiconducting polymer nanoparticles as photoacoustic molecular imaging probes in living mice. Nat Nanotechnol. 2014; 9: 233-9.

61. Kim C, Jeon M, Wang LV. Nonionizing photoacoustic cystography in vivo. Opt lett. 2011; 36: 3599-601

\section{Author biography}

Dr Changho Lee is currently a postdoctoral fellow at the Johns Hopkins University (JHU) since 2016. He have studied at the Kyungpook National University for Ph.D degree and researched at the University of Illinois Urbana-Champaign and the University at Buffalo, the State University of New York as a visiting scholar. Before joining the JHU, he worked as an assistant research professor and the postdoctoral fellow at Pohang University of Science and Technology (POSTECH) in Pohang, Republic of Korea since 2013. He focused on developing novel-biomedical imaging modalities such as optical coherence tomography, photoacoustic imaging, integrated optical imaging, molecular optical imaging, and intraoperative surgical imaging.

Dr Woosung Kwon received his B.S. in Chemical Engineering with a minor in Physics (2010) and Ph.D. in Chemical Engineering (2013) from Pohang University of Science and Technology (POSTECH). After postdoctoral research at Stanford
University, he joined the faculty of Sookmyung Women's University, where he is now serving as an assistant professor of chemical and biological engineering. His research interests include (1) development of functional, biocompatible nanomaterials for theragnostic and optoelectronic applications, (2) advancement of functionality of nanomaterials through atomic doping and molecular conjugation, and (3) study of the effect of nanomaterials on physiological biochemical processes in living organisms to address nanotoxicological issues.

Songeun Beack obtained her bachelor's degree from the department of Materials and Science Engineering, Pohang University of Science and Technology in 2011. She is currently a Ph. D. student under the supervision of Prof. Sei Kwang Hahn. Her research is centered on nanomedicine of hyaluronic acid derivatives using two-photon microscopy.

Donghyun Lee is a $\mathrm{PhD}$ candidate in the Creative IT Engineering Department. Donghyun was born in Busan, Korea, received his B.S. in Electriacl Engineering from POSTECH in 2014. Donghyun is interested in the optical coherence tomography (OCT) and photoacoustic microscopy (PAM).

Yoonsang Park received his B.S. in Chemical Engineering from Pohang University of Science and Technology (POSTECH) in 2014. He is currently pursuing his Ph.D. in Chemical Engineering at POSTECH. His research interests include synthesis of luminescent carbonaceous nanomaterials (carbon nanodots, graphene quantum dots, carbon nitrides, etc.) and their application in optoelectronics and bioimaging.

Dr Hyemin Kim earned her Ph. D. degree in Materials Science and Engineering from Pohang University of Science and Technology. Her research is centered on the development of smart drug delivery systems using hyaluronic acid derivatives.

Dr Sei Kwang Hahn is a professor (the former SeAh Young Chair Professor) in the Department of Materials Science and Engineering at POSTECH. He obtained his B.S., M.S., and Ph.D. in the Department of Chemical and Biomolecular Engineering at KAIST. After working at the LG Chemical group for 5 years, he did his post-doctoral research with Prof. Allan Hoffman at the University of Washington. After that, he worked at the Hoffman-La Roche group, Chugai Pharmaceutical Co. in Japan for 3 years. He was a visiting professor at the Harvard Medical School. He published more than 110 papers including Nature Photonics, Nature Communications, and Progress in Polymer Science.

Dr Shi-Woo Rhee received his Ph.D. in Chemical Engineering from Massachusetts Institute of 
Technology (MIT) in 1984. After two years of postdoctoral research at MIT, he joined the faculty of Pohang University of Science and Technology $($ POSTECH) in 1986. He currently holds a joint appointment in the Department of Chemical Engineering at POSTECH as the professor emeritus and in the Department of Chemical and Biological Engineering at Sookmyung Women's University as the chair professor. His current research interests are carbon materials for electrode applications, fluorescent carbon nanomaterials for optoelectronic devices, and materials for electronic and solar cell devices. He has published more than 200 peer-reviewed journal articles and edited two books in the field of materials engineering.

Dr Chulhong Kim studied for his Ph.D. degree and postdoctoral training at Washington University in St. Louis, St. Louis, Missouri under the supervision of Dr. Lihong V. Wang, Gene K. Beare Distinguished Professor (main advisor), Dr. Younan Xia, and Dr. Samuel Achilefu. He is currently an associate professor of Creative IT Engineering at Pohang University of Science and Technology (POSTECH) in Republic of Korea. Before he joined the department, he was an assistant professor of Biomedical Engineering at the University at Buffalo, the State University of New York from Aug 2010 to Jan 2013. He has currently served as an Editorial Board Member of Photoacoustics Journal and American Journal of Nuclear Medicine and Molecular Imaging, and a Guest Editor of Journal of Biomedical Optics, BioMed Research International, and IEEE Pulse Magazine. His research interests are the development of novel biomedical imaging techniques including photoacoustic tomography, ultrasound-modulated optical tomography, fluorescence imaging, ultrasound imaging, and laser speckle contrast imaging. 